\title{
Acoustic Measurements Using Common Computer Accessories: Do Try This at Home
}

\author{
Dale H. Litwhiler, Terrance D. Lovell \\ Penn State Berks-LehighValley College
}

\begin{abstract}
This paper presents some simple techniques for acoustic measurements using common, readily available computer accessories together with intuitive LabVIEWTM software. The methods used are designed to capture the interest of a wide range of engineering and science students. The speed of sound in air can be determined with good precision using only the sound card, microphone and speakers found with many personal computers (IBM or Mac). Using the computer's sound card under LabVIEW control, a sophisticated acoustic measurement system can be created. Appropriate sound signals (including music or other sound clips) are generated, transmitted, received and processed to determine the speed of sound. After the speed of sound is known, relative positions of the speakers and microphone can easily be determined for various geometries using similar techniques and algorithms. By using familiar hardware and sound clips from their favorite music, students are motivated to experiment with these techniques at home. The methods employed are rich in content for courses involving the areas of acoustics, signal processing, instrumentation and measurement or combinations of these areas. The hardware and software to implement these measurements and illustrative examples are presented in detail.
\end{abstract}

The speed of sound is used in a variety of distance and fluid level measurement applications. Usually, the transit time (time-of-flight) of an acoustic burst is measured and the distance is calculated using the speed of sound in the media of propagation (usually air). Students are usually referred to a table in the appendix of a textbook to find the value of this quantity. The speed of sound however is one of the few physical quantities that can actually be experimentally determined by the students with an acceptable degree of accuracy using equipment that they probably already have at home. This capability greatly empowers the students to explore the concepts on their own. ${ }^{1,2}$ A personal computer with a sound card, external speakers and an external microphone is all the equipment that is needed to perform these measurements. LabVIEW software, which is quite prevalent in engineering education, provides an excellent means of controlling the computer hardware and processing the acoustic signals.

Various experiments can be constructed to measure the speed of sound in air using common laboratory equipment such as signal generators and oscilloscopes together with speakers and microphones. Some of these experiments exploit the relationship between the wavelength, frequency and velocity of propagation of a sound wave: 


$$
\text { speed of sound }=(\text { wavelength }) \times(\text { frequency })
$$

Distance measurements of the wave crests are made while a known frequency tone is transmitted. The speed of sound is then calculated. ${ }^{3}$

Other experiments involve the measurement of the time of flight of reflections (echoes) from a surface at a known distance from the source and microphone. Here the outgoing and returning signals are usually captured with an oscilloscope and the time of flight is estimate by observing the displayed signals. Some have used computer hardware (microphone) and software (GoldWave $\mathbb{C}$ shareware audio processing application) to capture the sound of tapping or hand clapping and observing the echo signatures of the recorded signal. ${ }^{4}$

The method described here was used in an instrumentation laboratory class for third-year electromechanical engineering technology students at Penn State Berks - Lehigh Valley College. The experiments are based on time of flight measurements and are designed to demonstrate the roll of software in developing a sophisticated system with simple hardware. The signal processing techniques are similar to those used in radar and sonar echolocation systems. ${ }^{5}$ The software performs all of the measurements with no guesswork left to the user.

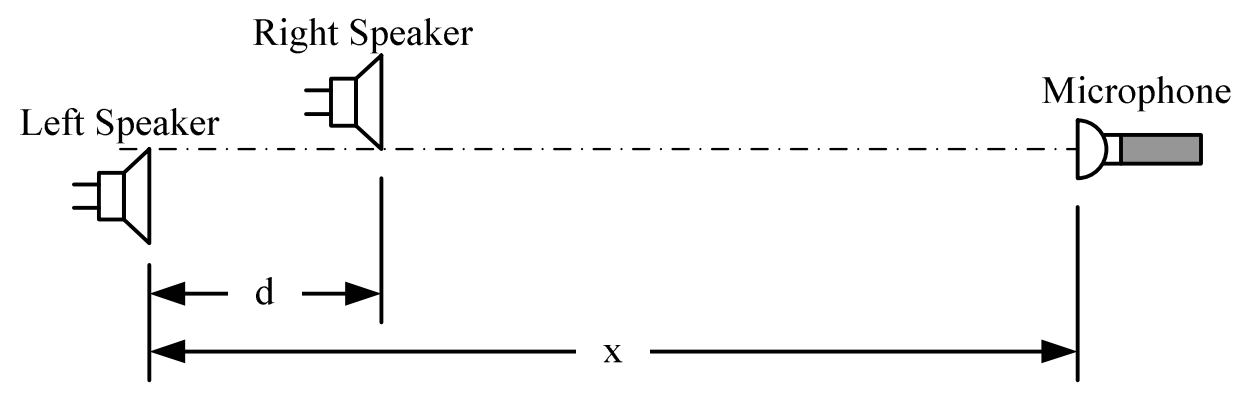

Figure 1. Setup for Acoustic Measurements

Using the computer's sound card under LabVIEW control, a brief audio burst containing unique left and right channel information is generated and played through the respective speakers. This audio burst is simultaneously recorded monaurally with the computer's sound card and microphone. Figure 1 shows the setup for the measurements. The recorded signal is processed using cross-correlation algorithms to determine the difference in arrival times of the audio channel signals. The geometry of the setup is then used to calculate the speed of sound in air. After the speed of sound is known, relative positions of the speakers and microphone can easily be determined for various geometries using similar techniques and algorithms. The audio bursts can consist of a variety of signals from simple chirps to CD-quality music clips.

\section{Chirp Signals}

It is important that the audio bursts transmitted through each channel can be uniquely identified in the received signal. In the context of signal processing, this requires that the autocorrelation of each channel's signal exhibit a distinct peak at a time shift of zero. Also, the cross-correlation 
of the channels' signals must not exhibit a distinct peak at any time shift value. So called chirp signals possess the desired autocorrelation qualities. ${ }^{6}$ A chirp signal has a constant amplitude while its frequency varies linearly over the duration of the signal. If the signal frequency increases with time, the signal is referred to as an up-chirp. Similarly, if the signal frequency decreases over the duration of the signal, a down-chirp is produced. Figure 2 shows the autocorrelation of an up-chirp signal with unity amplitude, zero mean, initial frequency of $770 \mathrm{~Hz}$, final frequency of $1477 \mathrm{~Hz}$ and duration of $50 \mathrm{~ms}$. The up-chirp signal is a sequence of discrete values with a sampling rate of $44.1 \mathrm{kHz}$ (2205 total samples). Figure 2 also shows the complementary down-chirp signal autocorrelation. Note that in these sequences, zero time shift corresponds to a sample shift of 2205 on the $\mathrm{x}$-axis.

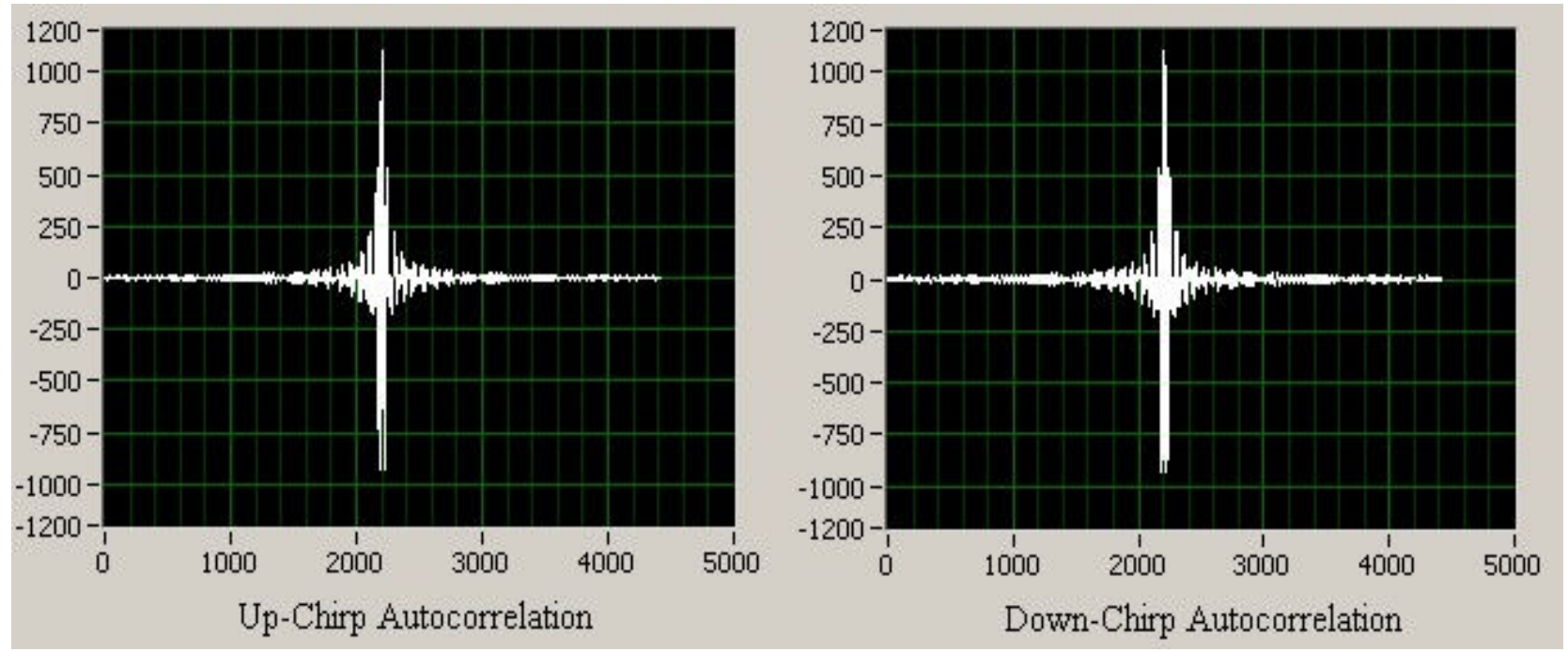

Figure 2. Up-Chirp and Down-Chirp Signal Autocorrelations

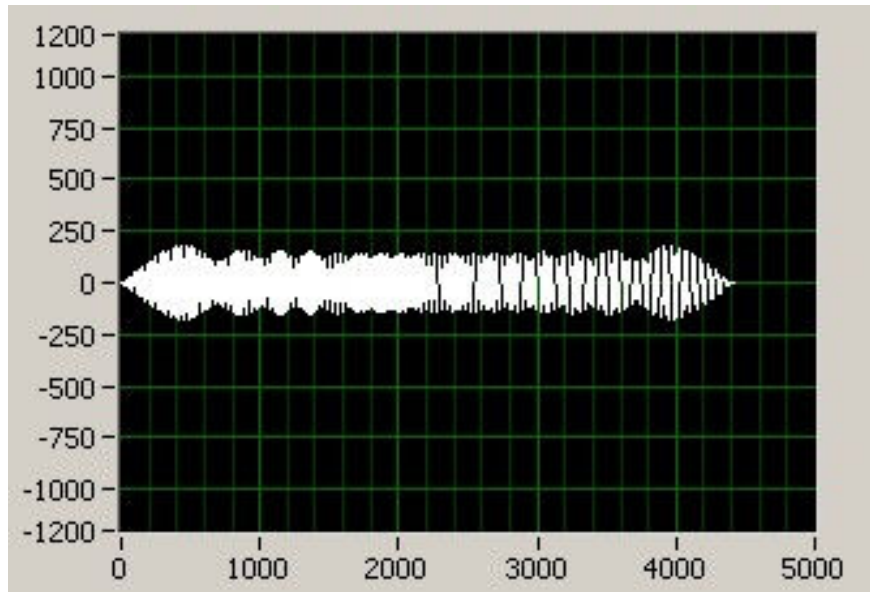

Figure 3. Cross-Correlation of the Up-chirp and Down-chirp Signals

Proceedings of the 2005 American Society for Engineering Education Annual Conference \& Exposition Copyright (C) 2005, American Society for Engineering Education 
Figure 3 shows the cross-correlation of the up-chirp and down-chirp signals described above. Notice that the same vertical scale is used for figures 2 and 3 to show the small relative amplitude of the cross-correlation compared to the autocorrelations. This quality is what makes the chirp a very useful signal for time-of-flight type of measurements. The transmitted signal can be found in a received signal record by performing a cross-correlation between the transmitted and received signals. The time (or sample number) of the peak of the crosscorrelation waveform indicates the amount by which the transmitted signal must be shifted in time (or samples) to line up with the received signal. The uniqueness of the chirp signal allows it to be located in a recorded signal containing large amounts of background noise.

\section{Sound Clip Signals}

Using similar ideas described as those described for chirps, it is possible to use other familiar sound clips for time of flight acoustic measurements. The motivation here is to allow the students to have some fun with the experiment and to learn about the nature and properties of sound signals. The sound clips can come from just about any source such as bits of music or movie soundtracks. The length of the clip and the sampling rate must be adjusted to keep the total size of the array within manageable limits.

When stereo music sound clips are analyzed, the cross-correlation of the left and right channels usually exhibits an undesirable central peak. This indicates that the channels have a lot of information in common which makes them not well suited for time of flight measurements. If however a monaural clip is split into two sequential sequences it is possible to generate a useful pair of sound bursts that are fairly uncorrelated. The first sequence is transmitted through the left channel followed immediately by the second sequence transmitted through the right channel. When the speakers are close together, the sound clip sounds nearly the same as the original full length sequence however when the recorded signal is analyzed, the difference in transit times of the two half sequences is apparent. From this difference, the speed of sound or the relative positions of the speakers can be calculated.

To help the students select an appropriate sound clip, a LabVIEW virtual instrument was developed. Figure 4 shows the front panel and Figure 5 shows the block diagram of this VI. Here the students can test different WAV file sound clips to see if they possess the appropriate autocorrelation properties for use in time of flight measurements. The VI prompts the user to select a sound file. The VI then performs the autocorrelation of the entire clip, splits the clip into two equal sequences, then performs the autocorrelation on each of them as well as their crosscorrelation. Based on the shape of the correlation plots, the students decide if the clip is worth trying in the time of flight experiments. As shown in Figure 5, the WAV file is processed to produce a 16-bit mono output sequence. If the original clip is in stereo format, the left and right channels are averaged point-for-point. Clips that are in 8-bit format, are converted to 16 bit format. The average value of each clip and sub-clip is subtracted to produce a zero-mean sequence prior to computing the correlations. (The sound clip used to create the waveforms in Figure 4 is the voice of actor Tom Hanks saying, "Houston, we have a problem," from the movie Apollo 13.) 


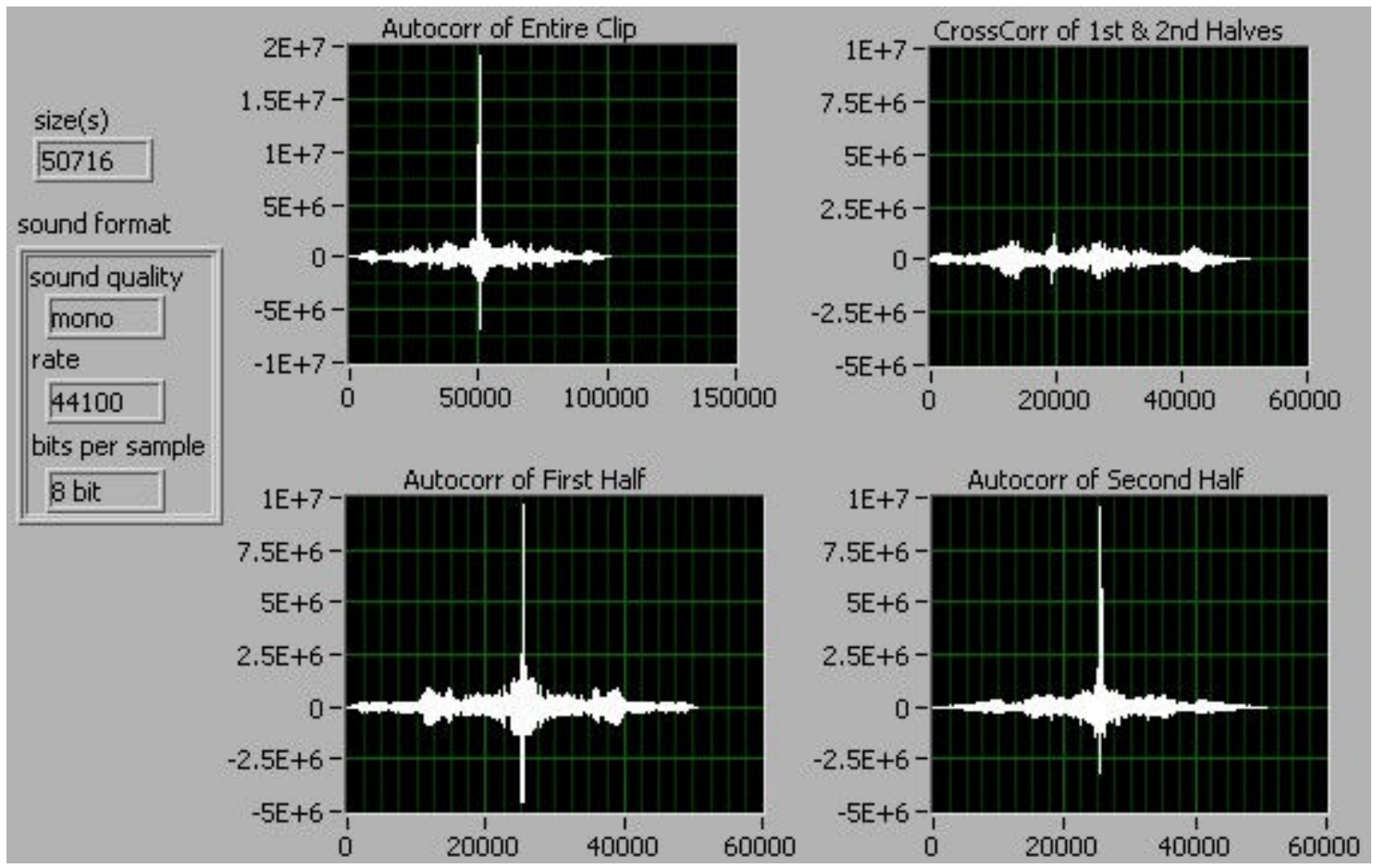

Figure 4. Front Panel of Sound Clip Correlation VI

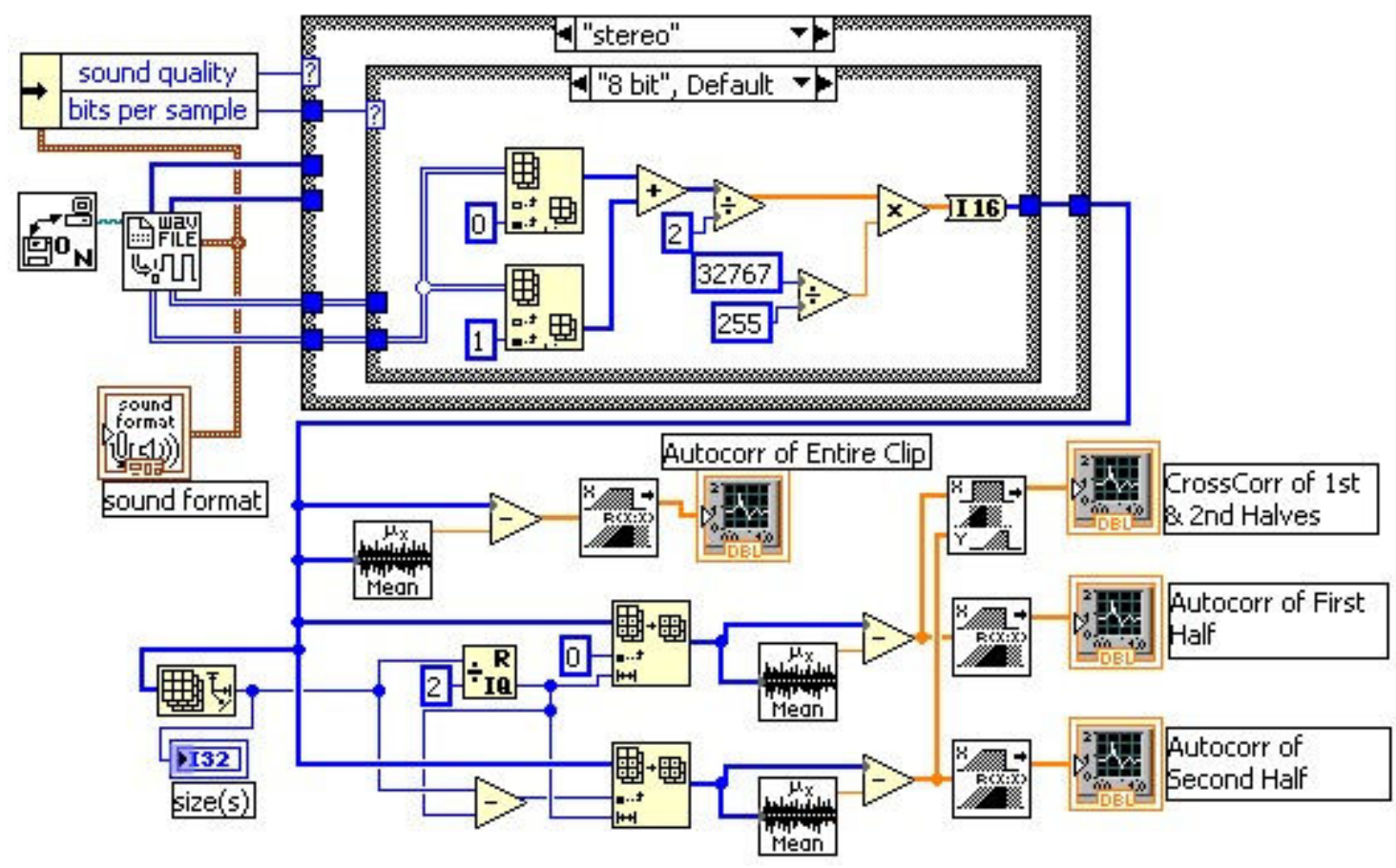

Figure 5. Block Diagram of Sound Clip Correlation VI

Proceedings of the 2005 American Society for Engineering Education Annual Conference \& Exposition Copyright ( 2005, American Society for Engineering Education 


\section{Experiment Examples}

\section{Measurements with Chirps}

Figure 6 shows the LabVIEW front panel of the virtual instrument for measuring the speed of sound (or the distance between speakers if the speed of sound is already known) using and upchirp and down-chirp signal. The computer speakers and microphone are arranged as previously shown in Figure 1. This is best accomplished on a bench top or on the floor. The distance of the microphone from the speakers is not critical but the students can also experiment with this. The spacing of the speakers, $d$, is measured and entered into the "Speaker Spacing (m)" control input of the VI. The user may choose to adjust the burst duration, chirp high tone frequency, chirp low tone frequency, and the sampling rate. When the VI is run and the toggle switch is in the "Speed of Sound" position, the calculated speed of sound will be displayed. The speed of sound is now known and the VI has been calibrated for use in measuring the speaker spacing.

To determine the speaker spacing, the user moves the toggle switch to the "Speaker Spacing" position. The value displayed in the "Speed of Sound $(\mathrm{m} / \mathrm{s})$ " indicator is now used to calculate the speaker spacing. The calculated value for the spacing is displayed in the "Speaker Spacing (m)" control location. This experiment can be repeated as the speaker spacing is varied along the axis line shown in Figure 1. In this mode, the speaker / microphone system becomes a location and tracking system.

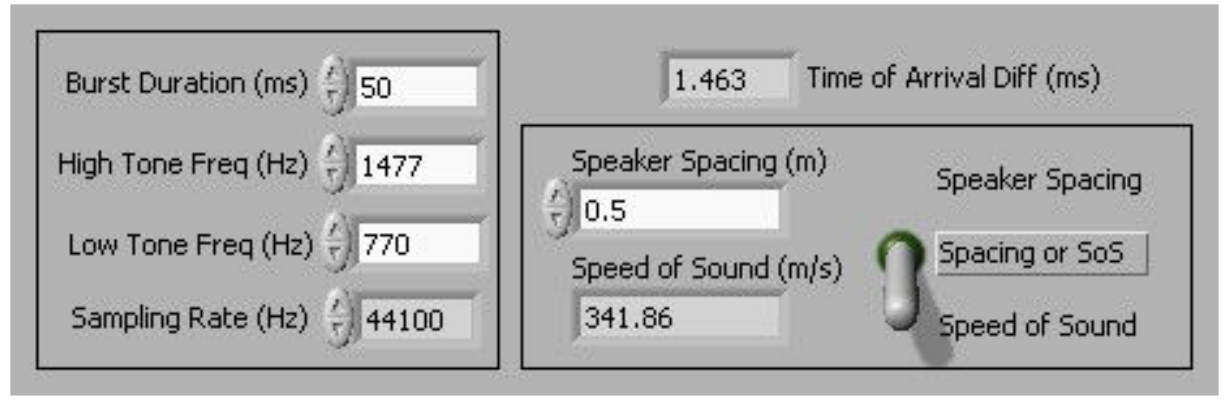

Figure 6. Speed of Sound / Speaker Spacing VI Front Panel

Figure 7 shows the LabVIEW block diagram for the speed of sound / speaker spacing virtual instrument. The sampling rate must be a standard value of $8000,11025,22050$, or $44100 \mathrm{~Hz}$. The "Create Up \& Down Chirp" subVI creates the chirp signal sequences by using the sample rate, burst duration, high tone frequency and low tone frequency values. The subVI then formats these sequences into an 8-bit stereo signal. The left channel contains the up-chirp signal and the right channel contains the down-chirp signal. This stereo signal is then passed to the "Send Sound and Record" subVI.

In the "Send Sound and Record" subVI the computer's sound card is set to begin recording the microphone input signal, the stereo chirp signal is sent to the speakers and the recorded signal sequence is retrieved. The retrieved sequence is in WAV file format. As described before, the average of the sequence must be removed prior to computing the correlations. 
The zero-mean recorded sequence is then cross-correlated with the up-chirp and down-chirp sequences that were generated in the "Create Up \& Down Chirp" subVI. The sequence index of the maximum value of each cross-correlation function is determined. Because the up-chirp and down-chirp sounds are sent simultaneously (the stereo signal), the difference between the crosscorrelation maximum indices is a measure of the time of arrival difference of the left and right channel chirps.

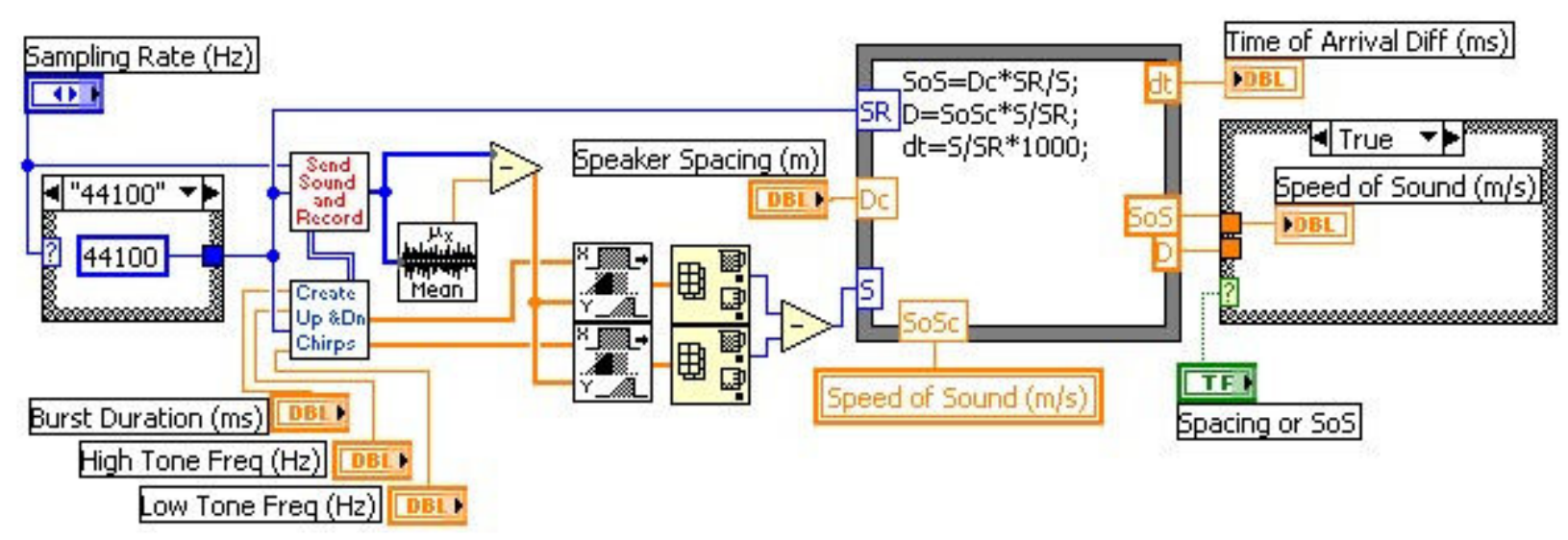

Figure 7. Speed of Sound / Speaker Spacing VI Block Diagram

The equation node shown in Figure 7 computes the speed of sound (SoS), the speaker spacing (D), and the time of arrival difference (dt). Depending on the mode of operation of the VI (set by the "Spacing or SoS" toggle switch), either the speed of sound or the speaker spacing output of from the equation node is displayed.

\section{Measurements with Sound Clips}

As discussed earlier, audio clips from music or any other common source possessing acceptable correlation characteristics can also be used in acoustic measurements. Although there is not necessarily a technical advantage to using sound clips, their use can be quite amusing and spark interest in engineering and science students. A LabVIEW virtual instrument has been developed in a similar manner as that for up-chirp and down-chirp sounds.

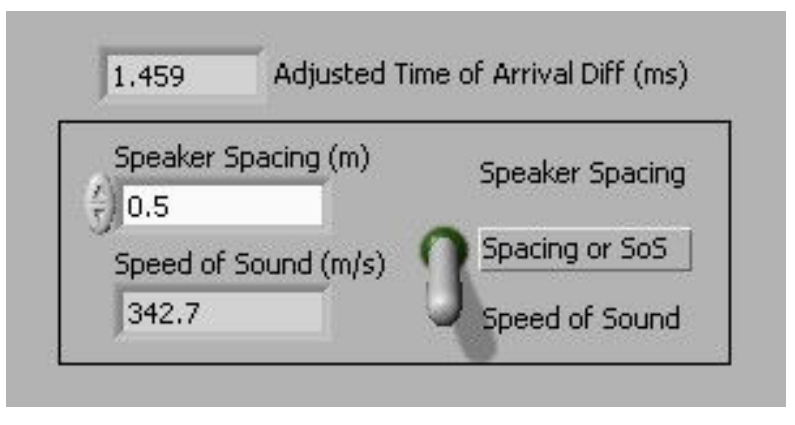

Figure 8. Acoustic Measurements with Sound Clip VI Front Panel 
Figure 8 shows the LabVIEW front panel for the VI to use a sound clip to determine the speed of sound or the distance between speakers for the equipment setup shown in Figure 1. Figure 9 shows the block diagram for this virtual instrument.

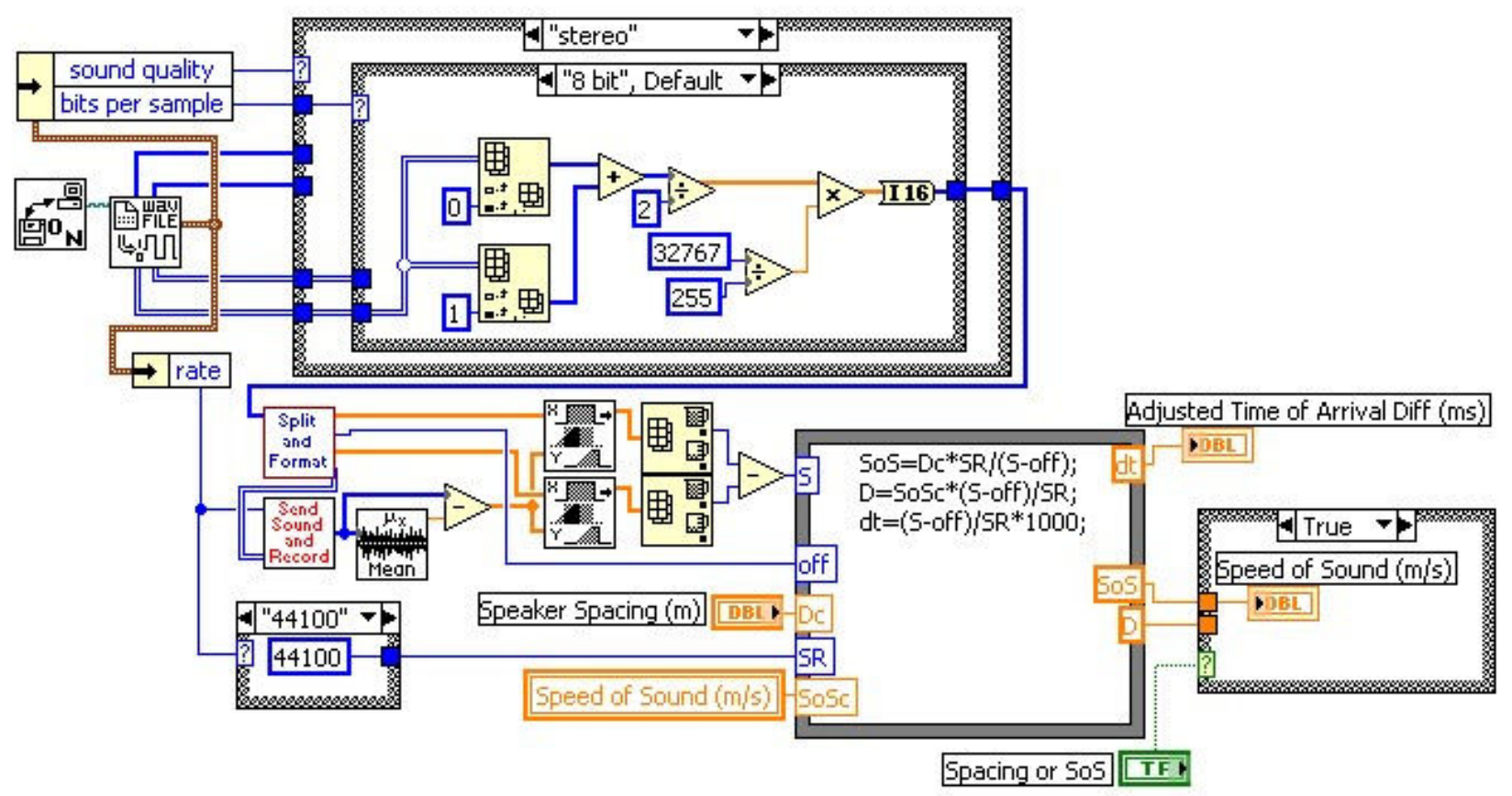

Figure 9. Acoustic Measurements with Sound Clip VI Block Diagram

When run, the VI prompts the user to select a file containing a suitable WAV formatted sound clip (This file was first tested using the VI shown in Figures 4 and 5). The sound clip parameters are evaluated (mono or stereo, 8 or 16 bit) and necessary adjustments are made to produce a 16bit monaural sequence. This sequence is split in half and reassembled by the "Split and Format" subVI to produce the required stereo signal as shown in Figure 10.

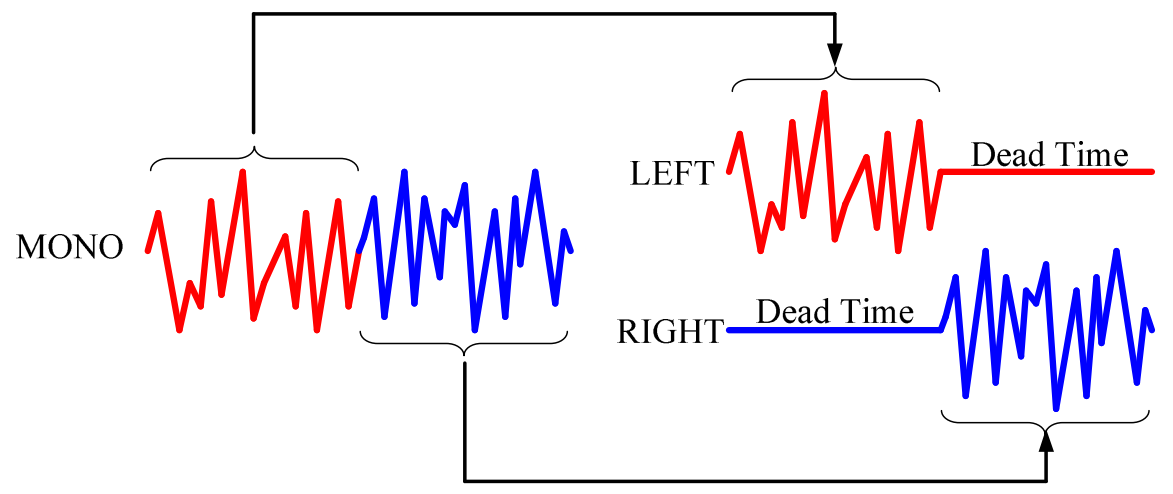

Figure 10. Sound Clip "Split and Format" Procedure 
The "Send Sound and Record" subVI the starts the recording of the microphone signal, sends the formatted stereo output signal to the speakers and retrieves the recorded sequence in WAV format. The average of the recorded sequence is removed prior to computing the correlations. The recorded signal is cross-correlated with both of the half-clip sequences. The index of the peak value of each of these cross-correlation functions is located and used to calculate the relative time of arrival of each channel's signal. Unlike the example with chirps, here the offset between the transmission times of the left and right channel must also be included in the time of arrival difference calculation. As with the chirp example, once the speed of sound has been determined, the VI can be used to determine the relative positions of the speakers for the geometry shown in Figure 1.

\section{Summary and Conclusions}

A sophisticated system for performing acoustic measurements with readily available computer hardware and software has been presented. The hardware consists of the stereo speakers and microphone supplied (or purchased for minimal cost) with most personal computers. The software used here is LabVIEW which is very popular in engineering and engineering technology programs. The student version of LabVIEW is available at very reasonable cost. The common hardware together with the student version of LabVIEW makes it possible for the acoustic measuring system presented here to be used by students at home. This capability allows the student to more freely experiment with the concepts of acoustic measurements which are then also applicable to many other areas. This is the main motivation behind this work.

Bibliography

1. Rogers, C., "Data Acquisition for the Dorm Room: Teaching Experimentation Techniques Using LEGO Materials," Proceedings of the American Society for Engineering Education Annual Conference and Exposition, 2001.

2. Braun, C. G., "Experiments on the Cheap: Using a Student Data Acquisition System," Proceedings of the American Society for Engineering Education Annual Conference and Exposition, 1997.

3. http://adelie.harvard.edu/ed/Activities/Speed_of_sound.html

4. http://www.sas.org/E-Bulletin/2003-11-14/features/index.html

5. Smith, Steven W., The Scientist and Engineer's Guide to Digital Signal Processing, $2^{\text {nd }}$ edition, California Technical Publishing, 1999, pp 137-140.

6. Dryer, Joseph E., "Improving Ultrasonic Transit Time Calculations,” Sensors, Vol. 21, No. 7, $2004,20-26$. 


\section{Biographies}

\section{DALE H. LITWHILER}

Dale H. Litwhiler is an Assistant Professor at Penn State Berks-Lehigh Valley College in Reading, PA. He received his B.S. from Penn State University (1984), his M.S. from Syracuse University (1989) and his Ph.D. from Lehigh University (2000) all in electrical engineering. Prior to beginning his academic career in 2002, he worked with IBM Federal Systems and Lockheed Martin Commercial Space Systems as a hardware and software design engineer.

\section{TERRANCE D. LOVELL}

Terrance D. Lovell is an electrical engineering student at Penn State Berks-Lehigh Valley College in Reading, PA where he is also completing his associate degree in electrical engineering technology. He serves as president of the student chapter of SAE and is also a laboratory assistant in the EET department. Prior to his academic pursuits he was an electronics countermeasures technician for the U.S. Marine Corps. 